\title{
A safe, stable, and convenient three- dimensional device for high Le Fort I osteotomy
}

Keisuke Sugahara ${ }^{1,2^{*}}$ (D), Masahide Koyachi ${ }^{1}$, Kento Odaka ${ }^{3}$, Satoru Matsunaga ${ }^{2,4}$ and Akira Katakura ${ }^{1,2}$

\begin{abstract}
Background: Le Fort I osteotomy is a highly effective treatment for skeletal jaw deformities and is commonly performed. High Le Fort I osteotomy is a modified surgical procedure performed for improving the depression of the cheeks by setting the osteotomy higher than the conventional Le Fort I osteotomy. Developments in threedimensional (3D) technology have popularized the use of 3D printers in various institutions, especially in orthognathic surgeries. In this study, we report a safe and inexpensive method of performing a high Le Fort I osteotomy using a novel 3D device and piezosurgery, which prevent tooth root injury without disturbing the operation field for patients with a short midface and long tooth roots.
\end{abstract}

Results: A 17-year-old woman presented with facial asymmetry, mandibular protrusion, a short midface, and long tooth roots. We planned high Le Fort I osteotomy and bilateral sagittal split ramus osteotomy. Prevention of damage to the roots of the teeth and the infraorbital nerve and accurate determination of the posterior osteotomy line were crucial for clinical success. Le Fort I osteotomy using 3D devices has been reported previously but were particularly large in size for this case. Additionally, setting the fixing screw of the device was difficult, because of the risk of damage to the roots of the teeth. Therefore, a different surgical technique, other than the conventional Le Fort I osteotomy and 3D device, was required. The left and right parts of the 3D device were fabricated separately, to prevent any interference in the surgical field. Further, the 3D device was designed to accurately cover the bone surface from the piriform aperture to the infra-zygomatic crest with two fixation points (the anterior nasal spine and the piriform aperture), which ensured stabilization of the 3D device. The device is thin and does not interfere with the surgical field. Safe and accurate surgical performance is possible using this device and piezosurgery. The roots of the teeth and the infraorbital nerve were unharmed during the surgery.

Conclusions: This device is considerably smaller than conventional devices and is a simple, low-cost, and efficient method for performing accurate high Le Fort I osteotomy.

Keywords: 3D device, Le Fort I osteotomy, Orthognathic surgery

\footnotetext{
* Correspondence: ksugahara@tdc.ac.jp

'Department of Oral Pathobiological Science and Surgery, Tokyo Dental

College, 2-9-18 Kanda Misaki-cho, Chiyoda-ku, Tokyo, Japan

${ }^{2}$ Oral Health Science Center, Tokyo Dental College, 2-9-18 Kanda Misaki-cho,

Chiyoda-ku, Tokyo, Japan

Full list of author information is available at the end of the article
}

\section{Springer Open}

(c) The Author(s). 2020 Open Access This article is licensed under a Creative Commons Attribution 4.0 International License, which permits use, sharing, adaptation, distribution and reproduction in any medium or format, as long as you give appropriate credit to the original author(s) and the source, provide a link to the Creative Commons licence, and indicate if changes were made. The images or other third party material in this article are included in the article's Creative Commons licence, unless indicated otherwise in a credit line to the material. If material is not included in the article's Creative Commons licence and your intended use is not permitted by statutory regulation or exceeds the permitted use, you will need to obtain permission directly from the copyright holder. To view a copy of this licence, visit http://creativecommons.org/licenses/by/4.0/. 


\section{Background}

The first description of a Le Fort I surgery was in the German language by Lamgenbeck in 1859 and in the USA by Cheever in 1864 for the resection of a nasopharyngeal tumor. This procedure was used to correct an open bite from a Guerin-type fracture in 1927, when Wassmund repositioned the maxilla without separating it from the pterygoid processes. Obwegeser developed the modern Le Fort I osteotomy procedure in which he completely immobilized the maxilla with the pterygomaxillary disjunction [1]. Conventional Le Fort I osteotomy can be successfully performed for patients with isolated maxillary hypoplasia, and the high Le Fort I osteotomy can be performed for patients with midfacial hypoplasia and a pronounced zygomatic deficiency. High Le Fort I osteotomy is a modified surgical procedure performed for improving the depression of the cheeks by setting the osteotomy higher than the conventional Le Fort I osteotomy [2]. The osteotomy line includes a part of the zygoma.

Developments in three-dimensional (3D) technology over the past decade have popularized the use of 3D printers in several institutions, especially in orthognathic surgeries. The "Fab Lab TDC" was the first digital fabrication laboratory for dentistry in Japan, which was established in 2013 [3]. Various 3D devices have been reported previously [3-5].

Here, we report a safe and inexpensive method of performing high Le Fort I osteotomy using a novel 3D device and piezosurgery.

\section{Materials and methods}

\section{Patient}

A 17-year-old woman presented with facial asymmetry, mandibular protrusion, short midface, and long tooth. We planned a high Le Fort I osteotomy and bilateral sagittal split ramus osteotomy.

\section{Method}

We designed the 3D device of the high Le Fort I osteotomy to prevent tooth root injury without disturbing the operation field for patients with a short midface and long tooth roots (Fig. 1).

\section{Results}

Prevention of damage to the roots of the teeth and the infraorbital nerve and accurate determination of the posterior osteotomy line was crucial for clinical success of the surgery. A method for accurate reproduction of virtually planned operations in Le Fort I osteotomy using 3D devices has been reported previously [5]. These 3D devices were stable but were particularly large in size for this case. Additionally, setting the fixing screw of the device was difficult, because of the risk of damage to the roots of the teeth. Therefore, a different surgical technique, other than the conventional Le Fort I osteotomy and $3 \mathrm{D}$ device, was required.

The computed tomography (CT) images of the patient were formatted as DICOM data, which were transferred to Minimics (Materialise, Leuven, Belgium) and 3-matic (Materialise, Leuven, Belgium), and set to allow the

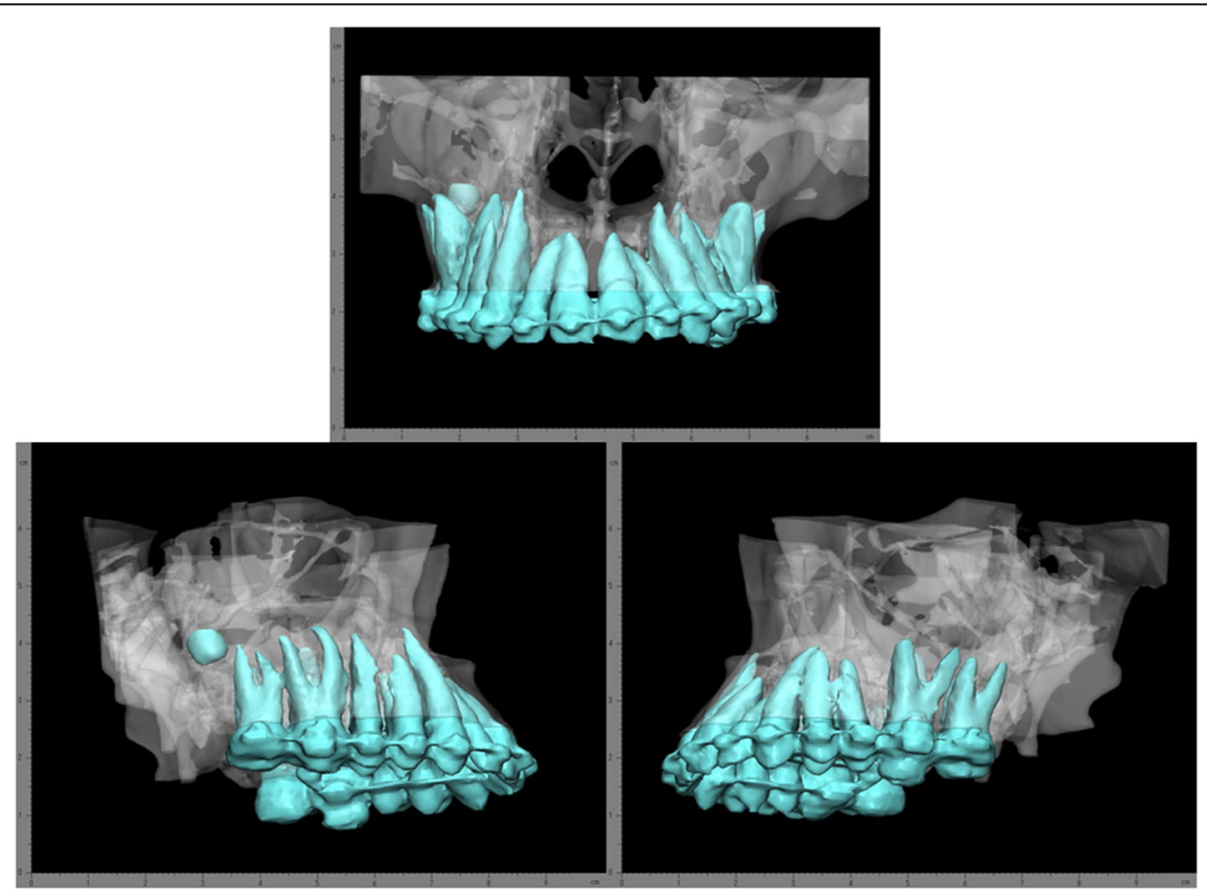

Fig. 1 Three-dimensionally reconstructed data showing the presence of long tooth roots and right maxillary third molar. (Front, Right, Left side view) 

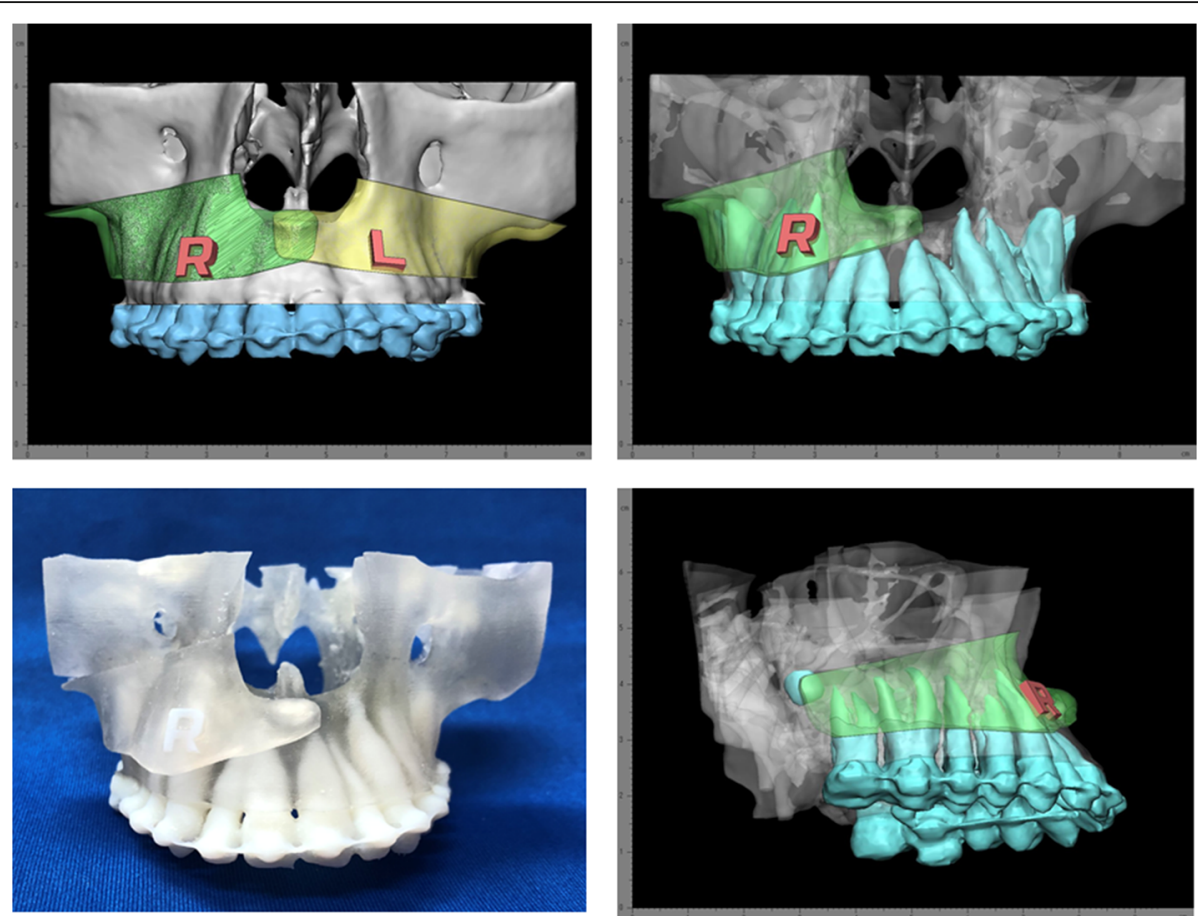

Fig. 2 Design of the three-dimensional device, which covers the anterior nasal spine and piriform aperture. The left and right parts of the 3D device were fabricated separately. We made a 3D model to verify the devices fit

virtual operation. The left and right parts of the 3D device were fabricated separately, to prevent any interference in the surgical field. Further, the 3D device was designed to accurately cover the bone surface from the piriform aperture to the infra-zygomatic crest with two fixation points (the anterior nasal spine and the piriform aperture), which ensured stabilization of the 3D device (Fig. 2). All data were converted to STL format, and the 3D device was fabricated using a 3D printer (Objet 260 Connex, Stratasys Ltd., Minnesota) in the Fab Lab TDC.

We chose the Neumann incision for this case, as it provides a sufficient field of view for the Le Fort I osteotomy; however, it leads to scarring in the maxillary vestibule (Figs. 3 and 4). Stability of the device during the procedure was ensured by maintaining complete contact of the 3D device with the bone surface instead of using a lateral incision.

\section{Discussion}

The 3D printing method is primarily derived from an additive manufacturing technology. 3D printing seems to have various applications in oral and maxillofacial surgery, particularly since the release of general use 3D printers on the market several years ago. 3D printing techniques have been used for corrective osteotomies, including orthognathic surgery, and have achieved some promising results in the last decade [6].

Globally, customized metal plates for jaw deformation and reconstruction are made with CAD/CAM based on preoperative computer simulations [7-9]. However, in Japan, only premade plates can be used in jaw deformity patients. As first fabrication laboratory for dentistry in Japan was established at Tokyo Dental College-the "Fab Lab TDC"-in December 2013 [1]. Techniques to construct full-scale 3D models, such as of the jaw, based on computed tomography $(\mathrm{CT})$ and magnetic resonance imaging (MRI) modalities have been reported recently [3-6].

We have also created preoperative 3D-printed models of cases for tumors in maxilla and mandible jaw

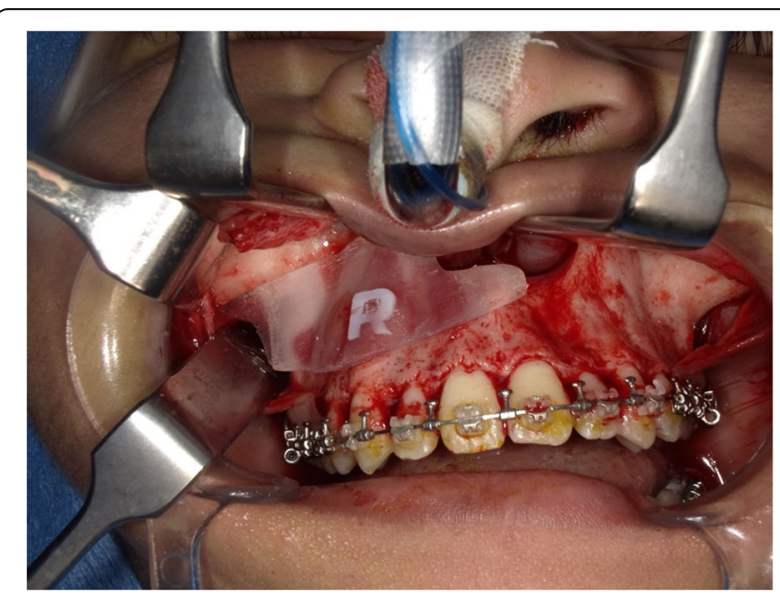

Fig. 3 Intraoperative view of the osteotomy using the threedimensional device (right side) 


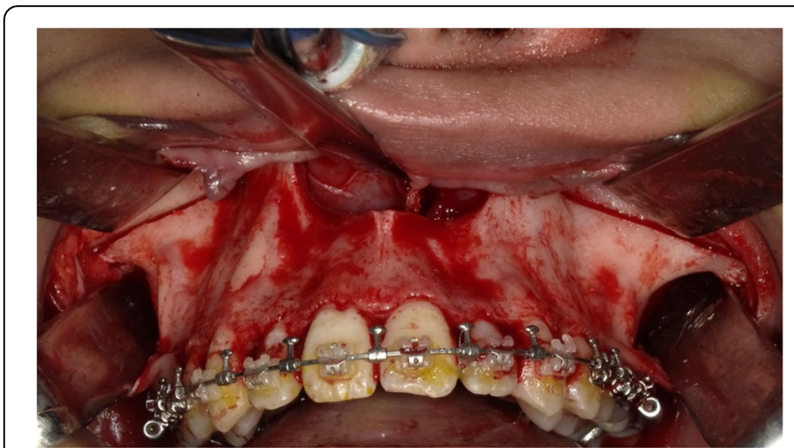

Fig. 4 Intraoperative view after the osteotomy. The roots of teeth are unharmed

deformities and used them primarily for consultations with patients and for preoperative simulations [3-5]. A method for accurate reproduction of virtually planned operations in the Le Fort I osteotomy using 3D devices has been reported previously [5]. These devices were stable but were large in size for this case. In addition, setting the fixing screw of the device was difficult, because of the risk of damage to the roots of the teeth. Therefore, a different surgical technique, other than the conventional Le Fort I osteotomy and 3D device, was required.

In the present study, we demonstrated that 3D devices manufactured using our design can be applied to perform safe, stable, and low-cost surgeries without the need for the fixing screw in patients with a short midface.

\section{Conclusion}

The device is thin and does not interfere with the surgical field. Safe and accurate performance of the surgery is possible using this device and piezosurgery. The roots of the teeth and infraorbital nerve were unharmed during the surgery.

This device is very economical and is a simple and efficient method for accurate Le Fort I osteotomy.

\section{Abbreviations}

3D: Three-dimensional; CAD/CAM: Computer aided design/computer aided manufacturing; CT: Computed tomography

\section{Acknowledgements}

We would like to thank the members of the Tokyo Dental College Suidobashi Hospital for their cooperation.

\section{Authors' contributions}

KS participated in the design of the study and coordination of the study. MK and $\mathrm{KO}$ worked in data collection and analysis. SM participated in the writing and helped to draft the manuscript. AK participated in the study design and correction of the manuscript and coordination. The authors read and approved the final manuscript.

\section{Authors' information}

KS is D.D.S., Ph.D., and FIBCSOMS.

\section{Funding}

This research was supported by grants for Private University Branding Project supported by the Ministry of Education, Culture, Sports, Science and
Technology, Japan, and Tokyo Dental College Branding Project for Multidisciplinary Research Center for Jaw Disease (MRCJD): Achieving Longevity and Sustainability by Comprehensive Reconstruction of Oral and Maxillofacial functions.

\section{Availability of data and materials \\ Not applicable}

Ethics approval and consent to participate

Ethics approval not required. The patients' permission was obtained.

\section{Consent for publication}

Not applicable

\section{Competing interests}

The authors declare that they have no competing interests.

\section{Author details}

'Department of Oral Pathobiological Science and Surgery, Tokyo Dental College, 2-9-18 Kanda Misaki-cho, Chiyoda-ku, Tokyo, Japan. ${ }^{2}$ Oral Health Science Center, Tokyo Dental College, 2-9-18 Kanda Misaki-cho, Chiyoda-ku, Tokyo, Japan. ${ }^{3}$ Department of Oral and Maxillofacial Radiology, Tokyo Dental College, 2-9-18 Kanda Misaki-cho, Chiyoda-ku, Tokyo, Japan. ${ }^{4}$ Department of Anatomy, Tokyo Dental College, 2-9-18 Kanda Misaki-cho, Chiyoda-ku, Tokyo, Japan.

Received: 20 July 2020 Accepted: 31 August 2020

Published online: 11 September 2020

\section{References}

1. Obwegeser H (2001) Mandibular growth anomalies terminology - aetiology diagnosis - treatment, vol 21. Springer, Berlin, Chapter, pp 385-415

2. Kim Yl, Park SB, Son WS, Hwang DS (2011) Midfacial soft-tissue changes after advancement of maxilla with Le Fort I osteotomy and mandibular setback surgery: comparison of conventional and high Le Fort I osteotomies by superimposition of cone-beam computed tomography volumes. J Oral Maxillofac Surg 69:e225-e233

3. Katsumi Y, Sugahara K, Matsunaga S, Odaka K, Mitomo K, Abe S et al (2016) Planning for orthognathic surgery at medical fabrication laboratory in Tokyo Dental College (Fab Lab TDC) clinical application of full-scale-model made by 3-dimensional ink jet printer for orthognathic surgery. Oral Science Japan 2016:9-11

4. Sugahara K, Katsumi Y, Koyachi M, Koyama Y, Matsunaga S, Odaka K et al (2018) Novel condylar repositioning method for 3D-printed models. Maxillofac Plast Reconstr Surg 40:4

5. Koyachi M, Sugahara K, Odaka K, Matsunaga S, Abe S, Sugimoto M et al The accuracy of Le Fort I osteotomy combined with computer-aided design/ computer-aided manufacturing technology and mixed reality. Int J Oral Maxillofac Surg (in press)

6. Lin HH, Lonic D, Lo LJ (2018) 3D printing in orthognathic surgery - a literature review. J Formos Med Assoc 117:547-558

7. Suojanen J, Leikola J, Stoor P (2016) The use of patient-specific implants in orthognathic surgery: a series of 32 maxillary osteotomy patients. J Craniomaxillofac Surg 44:1913-1916

8. Cornelius CP, Smolka W, Giessler GA, Wilde F, Probst FA (2015) Patientspecific reconstruction plates are the missing link in computer-assisted mandibular reconstruction: a showcase for technical description. J Craniomaxillofac Surg 43:624-629

9. Wilde F, Hanken H, Probst F, Schramm A, Heiland M, Cornelius CP (2015) Multicenter study on the use of patient-specific CAD/CAM reconstruction plates for mandibular reconstruction. Int I Comput Assist Radiol Surg 10: 2035-2051

\section{Publisher's Note}

Springer Nature remains neutral with regard to jurisdictional claims in published maps and institutional affiliations. 